\title{
Ocular albinism type 1-induced melanoma cell migration is mediated through the RAS/RAF/MEK/ERK signaling pathway
}

\author{
JUN BAI ${ }^{1}$, XIN XIE $^{2,3}$, YUN LEI $^{1}$, GAILI AN $^{1}$, LI HE$^{1}$ and XIAOPENG LV ${ }^{3}$ \\ ${ }^{1}$ Department of Medical Oncology, Shaanxi Provincial People's Hospital, The Third Affiliated Hospital of \\ The School of Medicine, Xi'an Jiaotong University, Xi'an, Shaanxi 710068; ${ }^{2}$ Key Laboratory of Resource Biology and \\ Biotechnology in Western China, Ministry of Education, College of Life Science, Northwest University, \\ Xi'an, Shaanxi 710069; ${ }^{3}$ Department of Translational Medicine, Institute of \\ Integrated Medical Information, Xi'an, Shaanxi 710016, P.R. China
}

Received September 7, 2013; Accepted March 4, 2014

DOI: $10.3892 / \mathrm{mmr} .2014 .2154$

\begin{abstract}
Malignant melanoma has the highest risk of mortality among all types of skin cancer due to its highly metastatic potential. The ocular albinism type $1(\mathrm{OA} 1)$ protein is a pigment cell-specific glycoprotein, which shares significant structural and functional features with $\mathrm{G}$ protein-coupled receptors. However, the role of OA1 in melanoma has yet to be elucidated. The present study aimed to investigate whether OA1 is involved in melanoma cell migration. OA1 was found to stimulate cell migration in a dose-dependent manner in cultured human melanoma cells. Furthermore, knockdown of OA1 using small interfering RNA was observed to significantly inhibit melanoma cell migration. In addition, the mechanism underlying OA1-induced melanoma cell migration was investigated. Stimulation of the RAS/RAF/mitogen activated protein kinase kinase (MEK)/extracellular signal-regulated kinase (ERK) pathway using growth factors enhanced OA1 expression and melanoma cell migration, whereas inhibition of this pathway using U0126 was observed to markedly decrease OA1 expression and the number of migrated cells. These findings indicate that OA1 is involved in melanoma cell migration and that OA1-induced melanoma cell migration is mediated through the RAS/RAF/MEK/ERK signaling pathway. Therefore, OA1 may serve as a novel therapeutic target for melanoma.
\end{abstract}

Correspondence to: Dr Jun Bai, Department of Medical Oncology, Shaanxi Provincial People's Hospital, The Third Affiliated Hospital of the School of Medicine, Xi'an Jiaotong University, 256 YouYi West Road, Xi'an, Shaanxi 710068, P.R. China

E-mail: junbaixian@163.com

Key words: ocular albinism type 1, melanoma, migration, $\mathrm{RAS} / \mathrm{RAF} /$ mitogen activated protein kinase kinase/extracellular signal-regulated kinase signaling pathway

\section{Introduction}

Melanoma is a highly aggressive and frequently chemoresistant type of skin cancer. In the United States, melanoma is the fifth and sixth most common type of skin cancer diagnosed in males and females, respectively (1). Patients with metastatic melanoma have a poor prognosis, with a five-year overall survival rate of $15 \%$ (2). Despite recent therapeutic advances in the management of melanoma, aside from early surgical resection, no therapeutic modality has been found to have a high likelihood of curative outcome (3). Therefore, further studies to elucidate the molecular mechanisms underlying melanoma cell metastasis are required to improve melanoma treatment.

The progression of melanoma from the non-metastatic melanoma phase, also termed the radial growth phase, to the metastatic phase, also termed the vertical growth phase, involves various alterations in the expression of genes, including protease activated receptor-1 (4), glycoprotein non-metastatic melanoma protein B (5), melanoma differentiation-associated gene-9 (6) and ocular albinism type 1 (OA1) (7). OA1 was originally identified as the 404 amino acid protein product of the gene responsible for the disorder, OA1, and was isolated by a classical positional cloning strategy from the distal short arm of the X chromosome (8). OA1 is a pigment cell-specific glycoprotein, which shares significant structural and functional features with G protein-coupled receptors (GPCRs). Previous studies have shown that OA1 ubiquitination is required for the targeting of OA1 to the intraluminal vesicles of multi-vesicular endosomes, thereby regulating the balance between OA1 downregulation and OA1 melanosome delivery (9). In a previous study, OA1-knockdown mouse melanocytes were not only found to exhibit a reduced number of melanosomes and presence of macromelanosomes, but also an abnormal distribution of melanosomes at the cell periphery (10). However, the role of OA1 in melanoma remains unclear.

The aim of the present study was to investigate the role of OA1 in melanoma, which was achieved by knocking down OA1 using small interfering (si)RNA. In addition, the present study aimed to provide insight into the mechanism underlying OA1-induced melanoma. 


\section{Materials and methods}

Materials. Anti-OA1, -ERK 1/2, -phosphorylated (p)-ERK1/2 and $-\beta$-actin antibodies were purchased from Invitrogen Life Technologies (Carlsbad, CA, USA). All other chemicals and reagents were purchased from Sigma-Aldrich (St. Louis, MO, USA) unless stated otherwise.

Cell culture. A375 human melanoma cells were purchased from The Cell Bank of Type Culture Collection of the Chinese Academy of Sciences (Shanghai, China). Cells were grown in Dulbecco's modified Eagle's medium (DMEM; Invitrogen Life Technologies) supplemented with $10 \%$ heat-inactivated fetal bovine serum (FBS) and antibiotics $(100 \mathrm{U} / \mathrm{ml}$ penicillin and $100 \mu \mathrm{g} / \mathrm{ml}$ streptomycin) in a humidified atmosphere of $5 \% \mathrm{CO}_{2}$ at $37^{\circ} \mathrm{C}$. A375 cells were detached using trypsin-EDTA $(0.05 \%$ trypsin).

Quantitative polymerase chain reaction $(q P C R)$ analysis. Total RNA was extracted from melanoma cells using an RNA Plus kit (Takara Bio Inc., Dalian, China). A total of $1 \mu \mathrm{g}$ total RNA per sample was used for comlementary (c)DNA synthesis using the QuantiTect Reverse Transcription kit (Qiagen, Valencia, CA, USA) according to the manufacturer's instructions. The PCR reaction contained cDNA, $0.1 \mathrm{nmol} / \mathrm{l}$ forward and reverse primer mix and SYBR ${ }^{\circledR}$ Green I (Invitrogen Life Technologies). qPCR analysis was performed using the 7300 RT-PCR System (Applied Biosystems, Inc., Foster City, CA, USA). The primer sequences used were as follows: Forward, 5'-CGG AGA TCG GCA GGACTGAGCAC-3' and reverse, 5'-ATA GTG GGG GAT GGC GTG GT-3' for human OA1; and forward, 5'-GAT CAT TGC TCC TCC TGA GC-3' and reverse, 5'-ACT CCT GCT TGC TGA TCC AC-3' for $\beta$-actin. Amplification included one stage of $2 \mathrm{~min}$ at $50^{\circ} \mathrm{C}$ and one stage of $2 \mathrm{~min}$ at $95^{\circ} \mathrm{C}$ followed by 40 cycles of $15 \mathrm{sec}$ at $95^{\circ} \mathrm{C}$ and $30 \mathrm{sec}$ at $60^{\circ} \mathrm{C}$. Data were analyzed using the 7300 RT-PCR System software and OA1 transcript levels were adjusted relative to those of the internal control $\beta$-actin.

Western blot analysis. Total protein extracts were prepared using radio-immunoprecipitation assay lysis buffer (Beyotime, Nantong, China) according to the manufacturer's instructions. The protein concentration in the cell lysates was assessed using a bicinchoninic acid protein assay kit (Beyotime). For western blot analysis, proteins lysates (30 $\mu \mathrm{g} / \mathrm{lane})$ were separated using SDS-PAGE and transferred onto polyvinylidene difluoride membranes (Whatman PLC, Brentford, UK). The membranes were blocked for $20 \mathrm{~min}$ at room temperature using the SuperBlock ${ }^{\circledR}$ T20 (TBS) Blocking Buffer. Subsequent to blocking, target proteins were probed with anti-OA1, -ERK1/2, -p-ERK $1 / 2$ and $-\beta$-actin primary antibodies overnight at $4^{\circ} \mathrm{C}$. The membranes were washed and incubated with horseradish peroxidase-conjugated secondary antibodies. Following washing, the sites of antibody binding were visualized via chemiluminescence (Boehringer Mannheim GmbH, Mannheim, Germany) according to the manufacturer's instructions and the expression of each protein relative to $\beta$-actin expression was analyzed.

Recombinant lentivirus construction and OAl siRNA transfection. The OA1 lentivirus was constructed and amplified according to the manufacturer's instructions (BD Biosciences, San Jose, CA, USA). The sense strand for the OA1 double-stranded siRNA was synthesized using the following sequence: 5'-GGA TAT GAA CCA CAC GGA A-3'. The OA1 non-targeting control siRNA sequence was as follows: 5'-AAT TCT CCG AAC GTG TCA CGT-3'. In vitro co-transfection was performed using Lipofectamine ${ }^{\mathrm{TM}} 2000$ according to the manufacturer's instructions.

Transwell migration assay. Migration assays were performed using melanoma cells transfected with siRNA targeting OA1 or scrambled siRNAs. Cells were analyzed using Transwell ${ }^{\circledR}$ cell culture chambers (Abcam PLC, Cambridge, UK). The lower face of the polycarbonate membrane $(8-\mu \mathrm{m}$ pore size) was coated with $0.25 \mathrm{mg}$ fibronectin overnight. Following trypsin treatment, melanoma cells were washed three times with phosphate-buffered saline and were resuspended in DMEM supplemented with $4 \%$ FBS. Melanoma cells $\left(2 \times 10^{5} / \mathrm{ml}\right.$ in $100 \mu \mathrm{l}$ medium) were added to the upper chamber and $600 \mu \mathrm{l}$ DMEM was added to the lower chamber. Subsequent to incubation in a humidified atmosphere of $5 \% \mathrm{CO}_{2}$ at $37^{\circ} \mathrm{C}$ for $12 \mathrm{~h}$, all non-migrated cells were removed from the upper chamber using a cotton swab. Migrated cells were fixed using methanol for $10 \mathrm{~min}$ at $4{ }^{\circ} \mathrm{C}$ and stained with $0.5 \%$ crystal violet solution for $30 \mathrm{~min}$. The number of cells per four high power fields was counted using a phase-contrast microscope (Leica, Wetzlar, Germany) in order to determine the average number of cells that had migrated. The number of cells was considered to represent migration activity.

Statistical analysis. Data are presented as the mean \pm standard deviation. Student's t-tests were performed for the comparison of two groups and one-way analysis of variance was used for multiple comparisons. $\mathrm{P}<0.05$ was considered to indicate a statistically significant difference.

\section{Results}

OA1 expression in melanoma cells. To assess the functional role of OA1, OA1 was overexpressed using lentivirus-mediated OA1-cDNA transfection in melanoma cells. As shown in Fig. 1, qPCR and western blot analyses revealed a significant upregulation of OA1 expression in melanoma cells following transfection with OA1-cDNA. OA1-cDNA transfection was observed to increase OA1 mRNA and protein levels to $162.3 \pm 3.9$ and $158.1 \pm 2.3 \%$ of the control, respectively $(\mathrm{P}<0.05)$. In addition, melanoma cells transfected with siRNA-OA1 showed a significant downregulation of OA1 expression, with siRNA-OA1 reducing OA1 mRNA and protein levels to $31.3 \pm 0.9$ and $21.1 \pm 2.3 \%$ of the control, respectively $(\mathrm{P}<0.05)$.

OA1 promotes melanoma cell migration. In the present study, OA1 was found to be expressed in melanoma cells and it was hypothesized that the migration of melanoma cells was associated with the development of melanoma. Therefore, the effect of OA1 on human metastatic melanoma cell motility was assessed using the Transwell migration assay. As shown in Fig. 2, Transwell migration assay revealed a dose-dependent increase in melanoma cell migration by OA1. The number of migrated melanoma cells was observed to increase with 
A

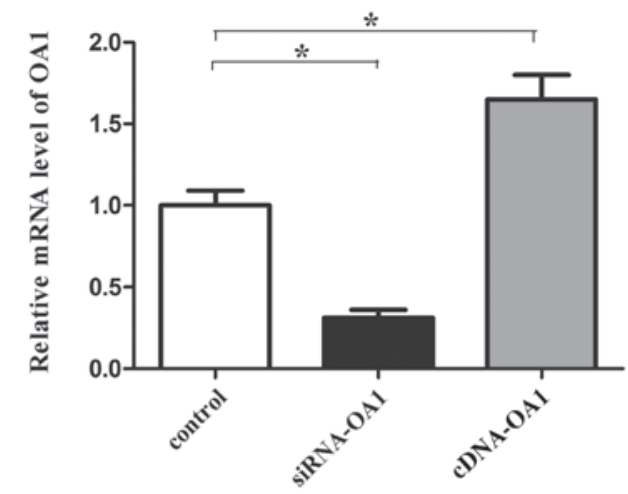

B

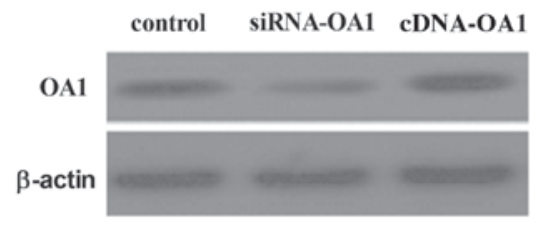

Figure 1. OA1 expression in melanoma cells. (A) Relative OA1 mRNA expression in A375 human melanoma cells transfected with cDNA-OA1 and siRNA-OA1. (B) Western blot analysis of A375 human melanoma cells transfected with cDNA-OA1 and siRNA-OA1. OA1 protein expression was normalized to that of $\beta$-actin. ${ }^{*} \mathrm{P}<0.05$ vs. the control. OA1, ocular albinism type 1; siRNA, small interfering RNA; cDNA, complementary DNA.

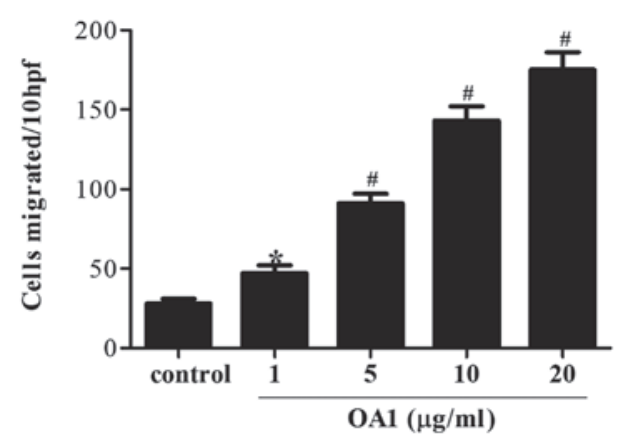

Figure 2. OA1 induces migration in melanoma cells. OA1 induced melanoma cell migration in a dose-dependent manner. Data are presented as the mean \pm standard deviation from three independent experiments. ${ }^{*} \mathrm{P}<0.05$ and ${ }^{\#} \mathrm{P}<0.01$ vs. the control. OA 1 , ocular albinism type 1 .

increasing OA1 concentration compared with the control group. Significant increases in cell migration were found to begin at $1 \mu \mathrm{g} / \mathrm{ml} \mathrm{OA} 1(\mathrm{P}<0.05)$ and peak at $20 \mu \mathrm{g} / \mathrm{ml} \mathrm{OA} 1$ $(\mathrm{P}<0.01)$. These findings indicate that $\mathrm{OA} 1$ may promote melanoma cell migration.

siRNA-induced OAl knockdown reduces melanoma cell migration. To further determine the function of OA 1 in promoting melanoma cell migration, a specific siRNA targeting OA1 was generated. As shown in Fig. 3, Transwell migration assay revealed that transfection with siRNA targeting OA1 significantly reduced the number of migrated cells, compared with transfection with scramble siRNA $(\mathrm{P}<0.05)$. Thus, OA1 may be involved in the regulation of melanoma cell migration.

OAl-induced melanoma cell migration is mediated by the RAS/RAF/MEK/ERK signaling pathway. The $\mathrm{RAS} / \mathrm{RAF} / \mathrm{MEK} / \mathrm{ERK}$ signaling pathway is important in the regulation of various cellular processes, including gene expression, proliferation, migration and survival (11-13). Therefore, the present study aimed to investigate whether OA1 was associated with the RAS/RAF/MEK/ERK signaling pathway. Two important stimulators of the RAS/RAF/MEK/ERK cascade were used: Epidermal growth factor (EGF) and

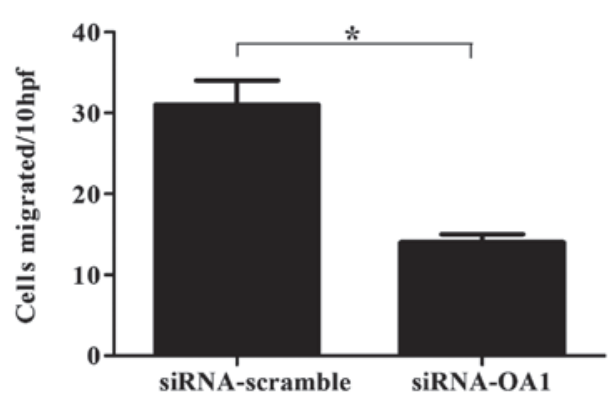

Figure 3. OA1 knockdown using siRNA attenuates A375 human melanoma cell migration. Transwell migration assay revealed that siRNA-OA1 significantly reduced the number of migrated cells compared with scramble siRNA. Results are presented as the mean \pm standard deviation from three independent experiments performed in duplicate. ${ }^{*} \mathrm{P}<0.05$ vs. siRNA-scramble. OA1, ocular albinism type 1; siRNA, small interfering RNA.

platelet-derived growth factor (PDGF). As shown in Fig. 4, the stimulation of ERK activity by EGF or PDGF was associated with an increase in OA1 protein expression and cell migration. To further investigate the role of RAS/RAF/MEK/ERK in OA1-induced melanoma cell migration, the effect of the RAS/RAF/MEK/ERK pathway inhibitor, U0126, on melanoma cell migration and OA1 expression was assessed. As shown in Fig. 5, U0126 markedly attenuated p-ERK1/2 and OA1 levels in melanoma cells. The cell migration rate was also significantly decreased by U0126. These findings indicate that OA1-induced melanoma cell migration is mediated by the RAS/RAF/MEK/ERK signaling pathway.

\section{Discussion}

Malignant melanoma has the highest risk of mortality among all types of skin cancer due to its highly metastatic potential. The incidence and mortality rates associated with malignant melanoma have continued to increase over recent years $(14,15)$. However, there is currently no effective treatment for metastatic melanoma, partly due to the complicated mechanism underlying its metastasis (16). In the present study, OA1 was found to promote the migration of melanoma cells in vitro. In addition, knockdown of OA1 using siRNA was 
A

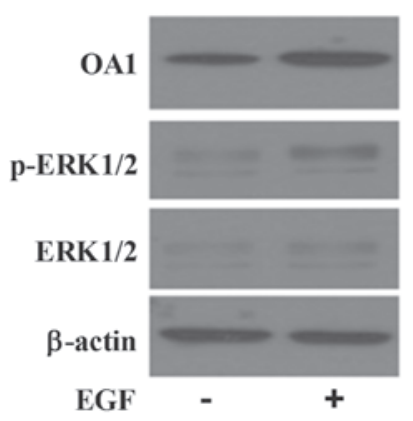

C

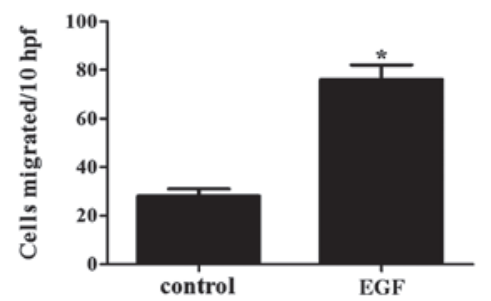

B

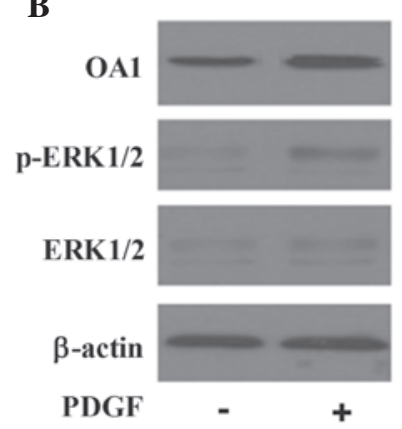

D

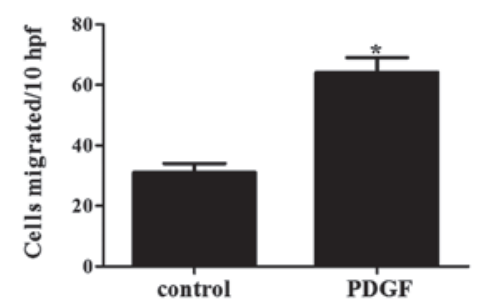

Figure 4. OA1 expression is upregulated through the ERK growth factor signaling pathway. A375 human melanoma cells were treated with 10 ng/ml EGF or PDGF for increasing durations. (A and B) Western blots analysis to detect OA1, p-ERK1/2 and ERK1/2 protein expression. (C and D) The number of migrated cells was significantly increased in response to ERK stimulation. "P<0.05 vs. the control. OA1, ocular albinism type 1; p-, phosphorylated; ERK, extracellular signal-regulated kinase; PDGF, platelet-derived growth factor; EGF, epidermal growth factor.

A

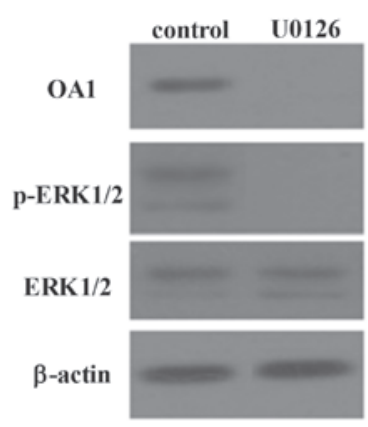

B

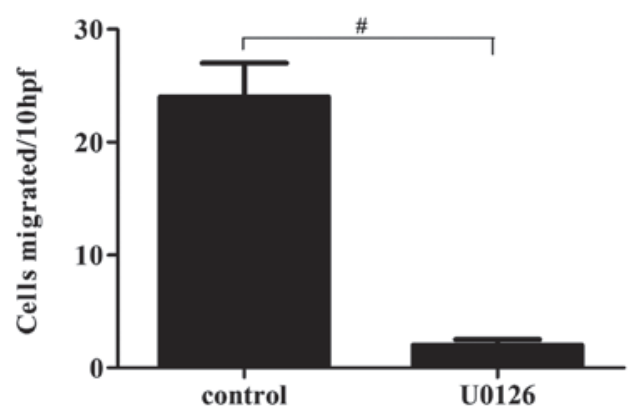

Figure 5. ERK inhibition inhibits OA1 expression and migration in A375 human melanoma cells. (A) OA1 and p-ERK1/2 protein expression decrease in response to U0126-induced ERK inhibition. (B) The number of migrated cells decrease in response to U0126-induced ERK inhibition. ${ }^{\#} \mathrm{P}<0.01$ vs. the control. OA1, ocular albinism type 1; p-, phosphorylated; ERK, extracellular signal-regulated kinase.

observed to inhibit melanoma cell migration. Furthermore, OA1-induced melanoma cell metastasis was found to involve the RAS/RAF/MEK/ERK signaling pathway. These findings may provide a novel target for melanoma treatment and may improve the future treatment of the disease.
The progression and metastasis of malignant melanoma are complex processes, which involve multiple cellular events, including cell proliferation, survival, migration and invasion. Metastasis is the spread of malignant tumor cells from a primary site to distant tissue and is the most life-threatening factor associated with cancer. A variety of metastasis-promoting and metastasis -suppressing genes have been identified to be involved in the metastasis of melanoma cells. Survivin is the smallest member of the inhibitor of apoptosis protein family (17). Certain studies have indicated that survivin enhances cell migration and the invasion of human melanoma cells (18). A previous report demonstrated that survivin promoted cell motility through activation of the protein kinase B signaling pathway and upregulation of $\alpha 5$ integrin (18). Furthermore, the survivin-mediated promotion of melanoma cell invasion was found to be dependent upon the activation of the mitogen-activated protein kinase pathway (18). Nm23-H1 was the first gene to be identified in a class of metastasis suppressor genes and the overexpression of Nm23-H1 in metastatic melanoma cells has been reported to reduce cell motility in vitro, as well as reduce metastatic potential in a xenograft model (19). In the present study, the effect of OA1 on human melanoma cell migration was investigated. OA1 was found to enhance melanoma cell migration in a dose-dependent manner. Moreover, siRNA-induced OA1 knockdown reduced melanoma cell migration. These findings indicate that OA1 may serve as a metastasis-promoting gene in melanoma development.

The RAS/RAF/MEK/ERK pathway has been reported to be activated in $>80 \%$ of all cutaneous melanomas, thus has been the focus of numerous investigations on melanoma (20). RAS/RAF/MEK/ERK signaling has been shown to promote cell proliferation, cell survival and tumor metastasis, as well 
as to be frequently aberrantly activated in cancer, in particular by the activation of upstream growth factors $(21,22)$. In the present study, the EGF and PDGF growth factors, which are important in melanoma development and progression, were found to stimulate ERK activity and increase OA1 expression. Inhibition of RAF and MEK kinase activities are the most investigated approaches for inhibiting ERK signaling $(23,24)$. RNA interference-mediated knockdown of oncogenic RAF has been reported to markedly reduce melanoma cell migration and ERK phosphorylation (25). In the present study, inhibition of the RAS/RAF/MEK/ERK signaling pathway using U0126 was found to decreases the levels of p-ERK1/2 and OA1 in melanoma cells. In addition, the number of migrated cells was decreased by U0126. Therefore, although the mechanism underlying the regulation of OA1 expression by the RAS/RAF/MEK/ERK signaling pathway has yet to be elucidated, the results of the present study indicate that OA1-induced melanoma cell migration depends on the RAS/RAF/MEK/ERK signaling pathway.

In conclusion, the present study demonstrated that OA1 is involved in the regulation of melanoma cell migration. Therefore, OA1 may be significant in human melanoma and may represent a novel therapeutic target for the prevention of melanoma.

\section{References}

1. Chakraborty R, Wieland CN and Comfere NI: Molecular targeted therapies in metastatic melanoma. Pharmgenomics Pers Med 6: 49-56, 2013.

2. Tas F: Metastatic behavior in melanoma: timing, pattern, survival, and influencing factors. J Oncol 2012: 647684, 2012.

3. Tsao H, Chin L, Garraway LA and Fisher DE: Melanoma: from mutations to medicine. Gene Dev 26: 1131-1155, 2012.

4. Zigler M, Kamiya T, Brantley EC, Villares GJ and Bar-Eli M PAR-1 and thrombin: the ties that bind the microenvironment to melanoma metastasis. Cancer Res 71: 6561-6566, 2011.

5. Li B, Castano AP, Hudson TE, Nowlin BT, Lin SL, Bonventre JV, Swanson KD and Duffield JS: The melanoma-associated transmembrane glycoprotein Gpnmb controls trafficking of cellular debris for degradation and is essential for tissue repair. FASEB J 24: 4767-4781, 2010

6. Das SK,Bhutia SK, Sokhi UK,AzabB,SuZZ,Boukerche H,Anwar T, Moen EL, Chatterjee D, Pellecchia M, Sarkar D and Fisher PB Raf kinase inhibitor RKIP inhibits MDA-9/syntenin-mediated metastasis in melanoma. Cancer Res 72: 6217-6226, 2012.

7. Fernandez LP, Milne RL, Pita G, Floristan U, Sendagorta E, Feito M, Avilés JA, Martin-Gonzalez M, Lázaro P, Benítez J and Ribas G: Pigmentation-related genes and their implication in malignant melanoma susceptibility. Exp Dermatol 18: 634-642, 2009.
8. Schiaffino MV: Signaling pathways in melanosome biogenesis and pathology. Int J Biochem Cell Biol 42: 1094-1104, 2010.

9. Giordano F, Simoes S and Raposo G: The ocular albinism type 1 (OA1) GPCR is ubiquitinated and its traffic requires endosomal sorting complex responsible for transport (ESCRT) function. Proc Natl Acad Sci USA 108: 11906-11911, 2011.

10. Palmisano I, Bagnato P, Palmigiano A, Innamorati G, Rotondo G, Altimare D, Venturi C, Sviderskaya EV, Piccirillo R, Coppola M, et al: The ocular albinism type 1 protein, an intracellular $\mathrm{G}$ protein-coupled receptor, regulates melanosome transport in pigment cells. Hum Mol Genet 17: 3487-3501, 2008.

11. Roberts PJ and Der CJ: Targeting the Raf-MEK-ERK mitogen-activated protein kinase cascade for the treatment of cancer. Oncogene 26: 3291-3310, 2007.

12. Hilger RA, Scheulen ME and Strumberg D: The Ras-Raf-MEK-ERK pathway in the treatment of cancer. Onkologie 25: 511-518, 2003.

13. Marshall C: Specificity of receptor tyrosine kinase signaling: transient versus sustained extracellular signal-regulated kinase activation. Cell 80: 179-185, 1995.

14. Bogenrieder T and Herlyn M: The molecular pathology of cutaneous melanoma. Cancer Biomark 9: 267-286, 2011.

15. Forsea A, Del Marmol V, de Vries E, Bailey EE and Geller AC: Melanoma incidence and mortality in Europe: new estimates, persistent disparities. Br J Dermatol 167: 1124-1130, 2012.

16. Bhatia S, Tykodi SS and Thompson JA: Treatment of metastatic melanoma: an overview. Oncology (Williston Park) 23: 488-496, 2009.

17. Ambrosini G, Adida C and Altieri DC: A novel anti-apoptosis gene, survivin, expressed in cancer and lymphoma. Nat Med 3: 917-921, 1997.

18. McKenzie JA, Liu T, Goodson AG and Grossman D: Survivin enhances motility of melanoma cells by supporting Akt activation and alpha5 integrin upregulation. Cancer Res 70: 7927-7937, 2010.

19. Marino N, Marshall JC and Steeg PS: Protein-protein interactions: a mechanism regulating the anti-metastatic properties of Nm23-H1. Naunyn Schmiedebergs Arch Pharmacol 384: 351-362, 2011.

20. Davies H, Bignell GR, Cox C, Stephens P, Edkins S, Clegg S, Teague J, Woffendin H, Garnett MJ, Bottomley W, et al: Mutations of the BRAF gene in human cancer. Nature 417: 949-954, 2002.

21. Agell N, Bachs O, Rocamora N and Villalonga P: Modulation of the Ras/Raf/MEK/ERK pathway by $\mathrm{Ca}^{2+}$, and Calmodulin. Cell Signal 14: 649-654, 2002.

22. Chang F, Steelman LS, Lee JT, Shelton JG, Navolanic PM, Blalock WL, Franklin RA and McCubrey JA: Signal transduction mediated by the Ras/Raf/MEK/ERK pathway from cytokine receptors to transcription factors: potential targeting for therapeutic intervention. Leukemia 17: 1263-1293, 2003.

23. Panka DJ, Wang W, Atkins MB and Mier JW: The Raf inhibitor BAY 43-9006 (Sorafenib) induces caspase-independent apoptosis in melanoma cells. Cancer Res 66: 1611-1619, 2006.

24. Sebolt-Leopold JS: MEK inhibitors: a therapeutic approach to targeting the Ras-MAP kinase pathway in tumors. Curr Pharm Des 10: 1907-1914, 2004.

25. Hingorani SR, Jacobetz MA, Robertson GP, Herlyn M and Tuveson DA: Suppression of BRAF(V599E) in human melanoma abrogates transformation. Cancer Res 63: 5198-5202, 2003. 